\title{
The effect of 3D scoliosis correction on the length of the spinal canal
}

\author{
Tom P. C. Schlösser, René M. Castelein \\ Department of Orthopaedic Surgery, University Medical Center Utrecht, Utrecht, The Netherlands \\ Correspondence to: Tom P. C. Schlösser, MD, PhD. Department of Orthopaedic Surgery, G05.228, University Medical Center Utrecht, P.O. Box \\ 85500, 3508 GA Utrecht, The Netherlands. Email: t.p.c.schlosser@umcutrecht.nl. \\ Provenance: This is an invited article commissioned by the Academic Editor Dr. Chenlong Yang (Department of Orthopedics, Peking University \\ Third Hospital, Beijing, China). \\ Comment on: Yahara Y, Seki S, Makino H, et al. Three-Dimensional Computed Tomography Analysis of Spinal Canal Length Increase After Surgery \\ for Adolescent Idiopathic Scoliosis: A Multicenter Study. J Bone Joint Surg Am 2019;101:48-55.
}

Submitted Nov 04, 2019. Accepted for publication Dec 02, 2019.

doi: $10.21037 /$ atm.2019.12.47

View this article at: http://dx.doi.org/10.21037/atm.2019.12.47

With great interest we read the study of Yahara et al. in which they investigated the three-dimensional (3D) length of the spinal canal from $\mathrm{T} 2$ to L2 in patient undergoing adolescent idiopathic scoliosis (AIS) surgery (1). Ultimately, they suggest that surgeons should understand how much the spinal canal is elongated by the deformity correction in order to lessen the risk of neurological complications. In this commentary, we try to link the findings of the present study to the current knowledge on the complex 3D pathoanatomy of AIS.

AIS is a classic orthopedic disorder that, regardless of its deeper underlying cause, is the result of an imbalance between the biomechanical loading of the upright human spine on the one hand and the body's compensating mechanisms on the other (2). Previous studies have shown that AIS is a rotational deformity, in which the anterior aspect of the apical area of the spinal deformity rotates away from the midline $(3,4)$. As already described by anatomists in the early 19th century, as well as more recently, using innovative $3 \mathrm{D}$ imaging techniques, by multiple research groups, it is now well known that this process of rotation away from the midline, in which the posterior structures stay behind, ultimately leads to the development of a rotated, apical lordosis of the apices of the scoliosis $(5,6)$. This lordosis, or excess anterior length, takes place predominantly in the soft tissues and not in the bone, there is no evidence of an actual disturbance of bony growth. Rather, the discs expand in their anterior aspects and the interlaminar and interspinous soft tissues are compressed posteriorly. Furthermore, this process occurs in other forms of scoliosis as well, it is by no means typical for AIS and should not be considered part of its etiology (7).

Thus, AIS is a complex $3 \mathrm{D}$ deformity of the spine, characterized by a segmental rotation, coronal deviation and a segmental lordosis of the apex of the spinal curvature. As a compensatory mechanism for global sagittal spinal balance, the junctional areas are relatively straight or even slightly kyphotic. On full-spine posterior-anterior radiographs in the standing position, most spines with AIS represent as a structural main thoracic curve, with a (non)structural compensatory high thoracic above and/or (thoraco) lumbar curve below. On lateral radiographs, the combination of the rotated segmental apical lordosis in combination with the alignment of the junctional zones may present as different global sagittal alignment patterns $(8,9)$.

In the present study, Yahara et al. conclude that corrective surgery for AIS elongates the spinal canal and that the preoperative coronal Cobb angles are predictors of postoperative spinal canal length after scoliosis surgery (1). Modern day surgical techniques for AIS aim to prevent for further spinal deformation and attempt to restore spinal balance. The basic principle for definitive AIS surgery implies that the deformity is corrected in all 3Ds, and the spine is fused in an optimally balanced configuration in all three planes (10). In all centers in this study derotational strategies were used. Theoretically this leads to coronal 
Cobb angle correction by derotation of the apical lordosis back to the midline. It the sagittal plane, however, it induces relative hypokyphosis and a higher inflection point. For recreation of physiological kyphosis, anterior shortening or posterior lengthening of the spine is inevitable. This corresponds to the finding that T2-L2 spinal length was iatrogenically increased by corrective surgery in all cases. Moreover, the larger the magnitude of the pre-operative $3 \mathrm{D}$ deformity and thus the pre-operative $3 \mathrm{D}$ rotated apical lordosis, the more posterior lengthening is necessary for successful sagittal restoration of the physiological double S-shape, especially at the apex. Therefore, the findings of increase of T2-L2 spinal length are the result of a combination of the pre-operative magnitude of the curves, the amount of derotation performed combined with the posterior lengthening procedure (active distraction and Ponte osteotomies).

Of course, the main goal of a surgical procedure for scoliosis, as for any other indication, is not to harm the patient. Neurological complications are devastating for the patient, the family, but also for the surgeon. So, any insight into the mechanisms that may put the patient at risk is welcome, and every attempt should be undertaken to avoid these complications. This includes state of the art neuromonitoring during the procedure to have an early warning system, that gives the opportunity to modify the surgical strategy and take appropriate measures when changes occur. Part of that strategy can be to reduce the amount of correction in whichever plane. Since we do not know the individual tolerance of the spinal cord to changes in length, the question remains, however, whether the surgeon should minimize beforehand this posterior elongation. 3D length of the spinal canal in the operated area of the spine may not be representative for the relative traction on the spinal cord by the deformity. First, the spinal cord has an excentric position in the concavity of the curve within the spinal canal in the apical area of AIS. Second, compensatory changes in spino-pelvic alignment in the nonfused area of the spine may also influence the elongation/traction of the spinal cord. Last years, there is increasing evidence that successful restoration of coronal and sagittal balance may have a protective effect on the unfused discs, as well as that it is important to re-create a critical thoracic kyphosis, in order to prevent flat back with further sagittal plane deterioration such as proximal or distal junctional kyphosis as well as decompensation of the cervical spine (11-14).

By combining the pathoanatomical data from previous studies with the data of the present study, we can conclude that (I) in order to correct the 3D spinal deformation, posterior lengthening of the spine, as well as anterior compression of the discs, is mandatory for restoration of spinal harmony in the sagittal plane, (II) for severe double thoracic curves, the posterior lengthening needed for adequate sagittal correction may reach the limit of stretch/ traction to what the spinal cord allows for without having severe neurophysiological complications. A potential alternative for posterior lengthening procedures may be anterior shortening by taking out a number of discs.

Although this study provides valuable information on the effects of modern day scoliosis surgery on the length of the spinal canal, it still remains unknown what the effect on the functioning of the spinal cord is As research continues on the $3 \mathrm{D}$ pathoanatomy of the spine and spinal canal in AIS, it can be expected that the effect of different surgical correction techniques on the length spinal canal will be revealed as well as the clinically relevant consequences for the spinal cord. Furthermore, 3D imaging modalities which allow for dynamic assessment of spine and spinal canal morphology (combined with neuromonitoring) in different body positions, may provide an opportunity to evaluate the limits of spinal cord stretch without neurological compromise, pre-operatively.

\section{Acknowledgments}

None.

\section{Footnote}

Conflicts of Interest: RM Castelein: unresitrcted K2M research grant. TPC Schlösser has no conflicts of interest to declare.

Ethical Statement: The authors are accountable for all aspects of the work in ensuring that questions related to the accuracy or integrity of any part of the work are appropriately investigated and resolved.

\section{References}

1. Yahara Y, Seki S, Makino H, et al. Three-Dimensional Computed Tomography Analysis of Spinal Canal Length Increase After Surgery for Adolescent Idiopathic Scoliosis: A Multicenter Study. J Bone Joint Surg Am 2019;101:48-55. 
2. Castelein RM, van Dieën JH, Smit TH. The role of dorsal shear forces in the pathogenesis of adolescent idiopathic scoliosis--a hypothesis. Med Hypotheses 2005;65:501-8.

3. Newton PO, Fujimori T, Doan J, et al. Defining the "Three-Dimensional Sagittal Plane" in Thoracic Adolescent Idiopathic Scoliosis. J Bone Joint Surg Am 2015;97:1694-701.

4. Schlösser TP, van Stralen M, Chu WC, et al. Anterior Overgrowth in Primary Curves, Compensatory Curves and Junctional Segments in Adolescent Idiopathic Scoliosis. PLoS One 2016;11:e0160267.

5. Nicoladoni C. Anatomie und Mechanismus der Skoliose. In: Kocher T, König P, von Mikulicz J, et al. editors. Bibliotheca Medica. Stuttgart: Verlag von Erwin Nagele, 1904.

6. Roaf R. The basic anatomy of scoliosis. J Bone Joint Surg Br 1966;48:786-92.

7. Brink RC, Schlösser TPC, Colo D, et al. Anterior Spinal Overgrowth Is the Result of the Scoliotic Mechanism and Is Located in the Disc. Spine (Phila Pa 1976) 2017;42:818-22.

8. Abelin-Genevois K, Sassi D, Verdun S, et al. Sagittal classification in adolescent idiopathic scoliosis: original description and therapeutic implications. Eur Spine J 2018;27:2192-202.

Cite this article as: Schlösser TPC, Castelein RM. The effect of $3 \mathrm{D}$ scoliosis correction on the length of the spinal canal. Ann Transl Med 2019;7(Suppl 8):S265. doi: 10.21037/ atm.2019.12.47
9. Post M, Verdun S, Roussouly P, et al. New sagittal classification of AIS: validation by $3 \mathrm{D}$ characterization. Eur Spine J 2019;28:551-8.

10. Shah SA. Derotation of the spine. Neurosurg Clin N Am 2007;18:339-45.

11. Nohara A, Kawakami N, Seki K, et al. The Effects of Spinal Fusion on Lumbar Disc Degeneration in Patients with Adolescent Idiopathic Scoliosis: A Minimum 10-Year Follow-Up. Spine Deform 2015;3:462-8.

12. Pasha S, Smith L, Sankar WN. Bone Remodeling and Disc Morphology in the Distal Unfused Spine After Spinal Fusion in Adolescent Idiopathic Scoliosis. Spine Deform 2019;7:746-53.

13. Shimizu T, Cerpa M, Lehman RA, et al. Reciprocal Change in Sagittal Profiles After Adolescent Idiopathic Scoliosis Surgery With Segmental Pedicle Screw Construct: A Full-body X-ray Analysis. Spine (Phila Pa 1976) 2019;44:1705-14.

14. Homans JF, Kruyt MC, Schlösser TPC, et al. Changes in the Position of the Junctional Vertebrae After Posterior Spinal Fusion in Adolescent Idiopathic Scoliosis: Implication in Risk Assessment of Proximal Junctional Kyphosis Development. J Pediatr Orthop 2019. [Epub ahead of print]. 